drastic impact on primitive cultures that has been attributed to it. But this is not necessarily true of more advanced societies, such as the classical civilizations of Central America and the Mediterranean. The effects of a huge eruption of Ilopango in El Salvador are shown to have had a profound effect on subsequent patterns of Mayan culture. The degree to which this was true of the eruption of Santorini in $1500 \mathrm{BC}$ is still the subject of controversy. Renfrew discusses evidence for and against the hypothesis that the eruption accounted for the final demise of Minoan civilization, and, using evidence that volcanologists may find less than conclusive, he concludes that the eruption and the destruction of the community on Crete, dated within 50 years of each other, were coincidental.

No book on the effect of volcanism on civilized communities would be complete without a chapter on the eruption of Vesuvius in $A D$ 79. Jashemski gives us a good summary of the eruption and its effects and explains some of the more recent archaeological work at Pompeii.

It is impossible to enumerate all the interesting facets of this volume. The editors deserve to be complimented on a splendid compilation of well-balanced papers, almost all of which are interesting, timely and well written. One of the most difficult tasks in a work such as this is keeping the papers on a level that will interest the average reader who is not concerned with details and the pet theories of individual specialists. The editors have managed to do this quite well. In short, the book is an unusually valuable contribution that will be useful to a wide range of readers.

Alexander R. McBirney is at the Centre for Volcanology, University of Oregon, and specializes in the study of the volcanoes of the circum-Pacific region. He is co-author of Volcanology, published last year by Freeman and Cooper.

\section{A star is born}

\section{F.D. Kahn}

Star Formation. 10th Advanced Course of the Swiss Society of Astronomy and Astrophysics. By I. Appenzeller, J. L. Lequeux and J. Silk. Pp.222. (Geneva Observatory: Sauverny, Switzerland, 1980.) SwFr.30.

THE brightest stars in spiral galaxies line the insides of the spiral arms. The same regions are rich in interstellar gas, much of it ionized and made luminous by the stars themselves, and concentrations of the dust tend to occur nearby. But the spiral arms are manifestations of a wave-like disturbance that travels at some $100 \mathrm{~km} \mathrm{~s}^{-1}$ relative to the material in the galactic disk. The bright stars must therefore be shortlived, and be born and evolve during the time that the star-forming phase of the wave passes through the region. Stellar structure theory confirms that such stars do indeed consume their nuclear fuel in a relatively short time; the most luminous do so in as little as three million years. All this has been known for two decades or so.

These observations suggest that the diffuse interstellar material provides the source of matter for the new stars, and various theories have been proposed to describe how this might happen. But the sequence of events must be complex: the mean mass density is typically 24 orders of magnitude lower in interstellar space than it is in a star. The condensation process will consequently have various stages, each dominated by a different set of physical parameters. In addition, formidable problems are posed by the angular momentum content of the interstellar gas, and by the magnetic flux linked with it. There must be dissipative processes to reduce them both, otherwise star formation would be completely inhibited.

Many new observations have been made in the past few years which bear on these questions. The spatial and kinematical structure of molecular clouds has been revealed by spectroscopic observations at millimetre wavelengths. These clouds invariably contain much dust; any stars that form within them are therefore enclosed at birth within a cocoon of dust. The stars thus become initially detectable as infra-red sources, since it is possible only to observe the radiation from the heated dust that surrounds them, and obscures their surfaces. It seems to take some half a million years for this dust to clear away.

The 1980 Advanced Course at Saas Fee was devoted to this range of topics. As always three lecturers took part, and it is clear that this year each one saw his task in a different way. The first set of talks (I. Appenzeller) describes how to use a computer to simulate the process of condensation of matter into a star. The calculations are reasonably tractable if spherical symmetry is assumed. But should the description not be full threedimensional? Can one even contemplate such a calculation for a system where some important lengths are as large as $10^{17} \mathrm{~cm}$, and others as small as $10^{12} \mathrm{~cm}$ ?

In the second group of talks, J. Lequeux sets out to establish some order among the large variety of observational data. $\mathrm{He}$ derives empirical formulae to connect the rate of star formation with the local density

of interstellar matter, and to describe the distribution of masses (the initial mass function or (MF) among the newly formed stars. But is there an IMF which applies everywhere? Can the IMF for massive stars be reconciled with that for the lighter stars?

In the last group of talks, J. Silk presents a wide-ranging survey, with no less than 157 references, of recent observational and theoretical results. It is easy to appreciate, after reading his contribution, how complex star formation really is, and how many physical interactions are involved. Yet even so he leaves the impression that an acceptable theory will evolve before long.

The Saas Fee courses have deservedly become famous. The present text maintains their high standard, and provides an excellent guide to a very important subject. The book is well produced and the price most reasonable. But would the organizers be willing to consider a small suggestion? In future, when the three lecturers have spoken, might it not be worthwhile to have another speaker to provide a summary and put the different views in perspective?

F. D. Kahn is Professor of Astronomy at the University of Manchester.

\section{A summary of 30 years of solar radio astronomy}

\section{R. T. Stewart}

Introduction to Solar Radio Astronomy and Radio Physics. By A. Kruger. Pp. 330. (Reidel: 1979.) Hardback Dfl.95, \$49.95; paperback Dfl.45, $\$ 23.50$.

THE last monograph on this subject appeared in 1964, and since then a great number of significant radio observations of the Sun have been made from space and from large ground-based arrays. Considerable progress has also been achieved in theoretical aspects of plasma emission processes. Thus, the appearance of this volume in which the author attempts to summarize the work carried out during the past 30 years, is timely.

The book is divided into five chapters. In Chapter 1 a short history is given of the early attempts to detect radio emission from the Sun, leading to its discovery in 1942. This is followed by a brief description of the general properties and structure of the Sun. Chapter 2 is devoted to various types of instruments and techniques used in observational solar radio astronomy.

The great variety of radio emissions from the Sun is the subject of the next chapter, which contains many good illustrations. Recent kilometric and hectometric radio observations from spacecraft 